\title{
Phonon Effects on the Weak Measurement of Charge States in Quantum Dots with a Quantum Point Contact
}

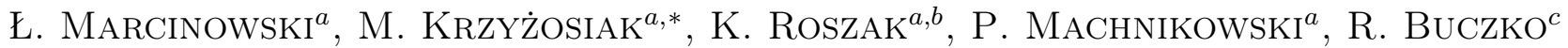 \\ AND J. MOSTOWSKI ${ }^{c}$ \\ ${ }^{a}$ Institute of Physics, Wrocław University of Technology, Wybrzeże Wyspiańskiego 27, 50-370 Wrocław, Poland \\ ${ }^{b}$ Department of Condensed Matter Physics, Faculty of Mathematics and Physics, Charles University \\ 12116 Prague, Czech Republic \\ ${ }^{c}$ Institute of Physics, Polish Academy of Sciences, al. Lotników 32/46, 02-668 Warsaw, Poland
}

\begin{abstract}
We study theoretically the process of a weak measurement of charge states in a double quantum dot performed by coupling to a quantum point contact. We focus on the interplay of the measurement process with phonon-induced dephasing of delocalized superpositions of electron states and compare the time scales relevant for the dynamics of the open system and for the measurement process: the decoherence rate, the localization rate of the state conditioned on the measurement outcome and the time scale on which information on the system state is gained in the process of the measurement.
\end{abstract}

PACS: 73.63.Kv, 03.65.Ta, 63.20.kd

\section{Introduction}

The measurement of charge and spin states in double quantum dots (DQDs) by monitoring the current through a quantum point contact (QPC) is commonly used in the most advanced experiments in the field $[1,2]$. The measurement is based on reading out the current through a constriction in a 2-dimensional electron gas (a QPC). The current depends on the external potential which makes the device sensitive to charges localized in the vicinity.

The dynamics of the DQD-QPC system has been extensively studied [3-9]. A series of tunneling events in the QPC constitutes a continuous measurement process in which the discrete current yields a growing amount of information on the system state which inevitably destroys the quantum coherence of the initial superposition and gradually localizes the initially delocalized electron in one of the dots according to the measurement outcome, consistently with the von Neumann projection principle. It has been shown [4] that the system can be described by a state vector at each instant of time if the measurement is "perfect" in the sense that each physical interaction with the measurement device leads to a unique detection trace. On the contrary, if some events are unobserved or if some physically distinguishable processes lead to identical detection events, one is forced to average over the unresolved possibilities and a description in terms of a density matrix is necessary.

An electron localized in a DQD structure undergoes

* current address: Beijing University of Technology, Pingleyuan 100, Chaoyang District, 100124 Beijing, China pure dephasing due to anharmonicity-induced phonon scattering on the polaron cloud surrounding the confined charge [10]. The characteristic time scales of this process are in the range of microseconds to milliseconds, comparable to the typical time scales over which the QPC current traces are observed [11, 12]. Therefore, it seems interesting to study the interplay of these two processes.

Here, we study theoretically the process of a weak measurement of single-electron charge states in a DQD performed by coupling to a QPC. We focus on the interplay of the measurement process with phonon-induced dephasing of delocalized superpositions of electron states. We develop a stochastic simulation method in the conditional density matrix formalism, based on the state-dependent waiting time distribution. We compare the three essential time scales relevant for the dynamics of the open system and for the measurement process: the decoherence rate, the localization rate of the state conditioned on the measurement outcome and the measurement time, defined as the time scale on which the measurement outcomes become statistically distinguishable. We show that the additional coupling to the phonon bath increases the decoherence rate but does not affect the time scales of the measurement process.

\section{The model}

We consider the DQD as a two-level system, in the basis of states representing one electron confined in the ground state of either the left or the right dot. The dots are identical and uncoupled. The confined electron interacts with the electronic bath formed by the 2 -dimensional 
electron gas in the source $(\mathrm{S})$ and drain (D) of the QPC as well as with the lattice vibrational modes (phonons). The Hamiltonian of the system is $H=H_{\mathrm{QPC}}+H_{\mathrm{ph}}$. The first contribution describes the electronic reservoir and its coupling to the QPC and has the form

$$
\begin{aligned}
& \mathcal{H}_{\mathrm{QPC}}=\sum_{k} \epsilon_{k}\left(a_{\mathrm{S}, k}^{\dagger} a_{\mathrm{S}, k}+a_{\mathrm{D}, k}^{\dagger} a_{\mathrm{D}, k}\right) \\
& +\sum_{k, q}\left(T_{k, k^{\prime}}+\chi_{k, k^{\prime}} \sigma_{z}\right) a_{\mathrm{D}, \mathrm{k}^{\prime}}^{\dagger} a_{\mathrm{S}, k}+\text { H.c. },
\end{aligned}
$$

where $a_{i, k}^{\dagger}$ and $a_{i, k}$ are the creation and annihilation operators for electrons, with $i=\mathrm{S}, \mathrm{D}$ labeling the part of the reservoir and $k$ denoting electron states, $T_{k, k^{\prime}}$ denotes the contribution to the amplitude for the tunneling through the QPC independent of the DQD state, and $\chi_{k, k^{\prime}}$ is the contribution depending on the state via the DQD-related operator $\sigma_{z}$ (defined in the basis defined above). The second part of the Hamiltonian,

$$
\mathcal{H}_{\mathrm{ph}}=\sum_{\boldsymbol{q}} \hbar \omega_{\boldsymbol{q}} b_{\boldsymbol{q}}^{\dagger} b_{\boldsymbol{q}}+\left(\sigma_{z} \sum_{\boldsymbol{k}, \boldsymbol{q}} F_{\boldsymbol{q}, \boldsymbol{q}^{\prime}} b_{\boldsymbol{q}}^{\dagger} b_{\boldsymbol{q}^{\prime}}+\text { H.c. }\right)
$$

describes the phonons and their coupling to the confined charge via a phonon scattering process [10]. Here $b_{\boldsymbol{q}}^{\dagger}$ and $b_{\boldsymbol{q}}$ are creation and annihilation operators for a phonon in the mode $\boldsymbol{q}$ and $\omega_{\boldsymbol{q}}$ is the corresponding frequency.

\section{The method of stochastic simulation}

Following Refs. [3] and [10], we write down the quantum Master (Lindblad) equation describing the evolution of the DQD system interacting with both reservoirs,

$$
\dot{\rho}=\sum_{i=0}^{2}\left[C_{i} \rho C_{i}^{\dagger}-\frac{1}{2}\left\{\rho, C_{i}^{\dagger} C_{i}\right\}\right],
$$

where $C_{i}=\gamma_{\mathrm{ph}}|i\rangle\langle i|, i=1,2$, are phonon-related Lindblad operators representing phonon scattering from the electron in the dot 1 and 2 , respectively, $C_{0}=\gamma_{a} \sigma_{z}+\gamma_{b} \mathbb{I}$ are operators representing the electron tunneling events in the QPC (II is the unit operator), $\gamma_{a} \pm \gamma_{b}$ is the probability amplitude for tunneling through the QPC with the electron in the right/left dot, and $\gamma_{\mathrm{ph}}$ is the probability amplitude for phonon scattering. This description is obtained via a number of approximations, including the short memory assumption (the Markov limit) for both processes and independence of the tunneling amplitudes of the initial and final electron states within the relevant energy range (see Refs. [10, 13]).

While Eq. (1) describes the system dynamics averaged over an ensemble (or a large number of repetitions), modern experimental techniques allow one to observe a single system history which starts, say, from an equally weighted superposition state and ends up in a projected, localized state. In order to model such a physical process one "unravels" the Lindblad equation [14], that is, represents the evolution as a sequence of deterministic flows interspersed with discontinuities (jumps) associated with the system-environment interaction events (tunneling through QPC and phonon scattering). Formally, the system evolution in each time step $\mathrm{d} t$ is governed (conditioned) by increments of the Poisson processes $\mathrm{d} N_{i}$ corresponding to the occurrence of the interaction or measurement events, with $\mathrm{d} N_{i} \mathrm{~d} N_{j}=\mathrm{d} N_{i} \delta_{i j}, p\left(\mathrm{~d} N_{i}=1\right)=\operatorname{Tr}\left(C_{i}^{\dagger} C_{i} \rho\right) \mathrm{d} t$ $[7,13]$. Depending on the value of the increments, the system either undergoes a deterministic evolution driven by the effective Hamiltonian $H_{\mathrm{eff}}=-(\mathrm{i} \hbar / 2) \sum_{i} C_{i}^{\dagger} C_{i}$ or jumps to a new state,

$$
\rho \rightarrow \begin{cases}\frac{C_{i} \rho C_{i}^{\dagger} \mathrm{d} t}{p\left(\mathrm{~d} N_{i}=1\right)}, & \mathrm{d} N_{i}=1, \\ \frac{\rho-(\mathrm{i} / \hbar) \mathrm{d} t\left[H_{\text {eff }}, \rho\right]_{*}}{1-\sum_{i} p\left(\mathrm{~d} N_{i}=1\right)}, & 1-\sum_{i} \mathrm{~d} N_{i}=1,\end{cases}
$$

where $\left[H_{\mathrm{eff}}, \rho\right]_{*}=H_{\mathrm{eff}} \rho-\rho H_{\mathrm{eff}}^{\dagger}$. Thus, the density matrix at the time $t+\mathrm{d} t$ can formally be written as

$$
\begin{aligned}
& \rho(t+\mathrm{d} t)=\sum_{i} \frac{C_{i} \rho C_{i}^{\dagger} \mathrm{d} t}{p\left(\mathrm{~d} N_{i}=1\right)} \mathrm{d} N_{i} \\
& +\frac{\rho-(\mathrm{i} / \hbar) \mathrm{d} t\left[H_{\mathrm{eff}}, \rho\right]_{*}}{1-\sum_{i} p\left(\mathrm{~d} N_{i}=1\right)}\left(1-\sum_{i} \mathrm{~d} N_{i}\right) .
\end{aligned}
$$

This equation represents the state of the system based on the observer's knowledge on all the interaction events. Let us note that if $\rho(t)$ is a projector, then $\rho(t+\mathrm{d} t)$ is a projector, too, and the system state might well be represented by a state vector. However, only the current flow is actually associated with an observed detection event, while the phonon scattering is not. Therefore, one has to average (mix) the conditional states corresponding to $\mathrm{d} N_{0}=0$ with weights equal to the relative (conditional) probabilities of their occurrence. As a result, one finds

$$
\begin{gathered}
\left.\rho(t+\mathrm{d} t)\right|_{\mathrm{d} N_{0}=0} \approx\left[1+\operatorname{Tr}\left(\rho(t) C_{0}^{\dagger} C_{0}\right) \mathrm{d} t\right] \rho \\
-\frac{\mathrm{i}}{\hbar} \mathrm{d} t\left[H_{\mathrm{eff}}^{\prime}, \rho\right]_{*}+\mathcal{L}_{i}[\rho] \mathrm{d} t,
\end{gathered}
$$

where $\mathcal{L}_{\mathrm{ph}}[\rho]=\sum_{i}\left[C_{i} \rho C_{i}-(1 / 2)\left\{C_{i}^{\dagger} C_{i}, \rho\right\}\right]$ is the phonon-related Lindblad dissipator, $H_{\text {eff }}^{\prime}=$ $-(\mathrm{i} \hbar / 2) C_{0}^{\dagger} C_{0}$ and the terms of higher order in $\mathrm{d} t$ were neglected. Therefore, in this case we have for the density matrix conditioned on the current flow events

$$
\begin{aligned}
& \rho(t+\mathrm{d} t)=\frac{C_{0} \rho C_{0}^{\dagger} \mathrm{d} t}{p\left(\mathrm{~d} N_{0}=1\right)} \mathrm{d} N_{0} \\
& +\frac{\rho-(\mathrm{i} / \hbar) \mathrm{d} t\left[H_{\text {eff }}^{\prime}, \rho\right]_{*}+\mathcal{L}_{i}[\rho] \mathrm{d} t}{1-p\left(\mathrm{~d} N_{0}=1\right)}\left(1-\mathrm{d} N_{0}\right) .
\end{aligned}
$$

For the simulation, we propose a method based on the waiting time distribution, which generalizes the state vector formalism described in Ref. [14]. This allows us to avoid the possible numerical difficulties related to the simple forward (Euler) iteration over the deterministic periods and use the available analytical form of the deter- 
ministic evolution. One can prove two statements: first, if $\tilde{\rho}$ evolves according to the deterministic equation without normalization,

$$
\left.\frac{\mathrm{d}}{\mathrm{d} t} \tilde{\rho}\right|_{\mathrm{det}}=-\frac{\mathrm{i}}{\hbar}\left[H_{\mathrm{eff}}^{\prime}, \tilde{\rho}\right]_{*}+\mathcal{L}_{i}[\tilde{\rho}]
$$

then $1-\operatorname{Tr}(\tilde{\rho})$ is the cumulative distribution function (CDF) for the waiting time (the time until the next jump). Second, the normalized density matrix for a deterministic evolution is obtained from $\tilde{\rho}$ by normalization, $\rho_{\text {det }}(t)=\tilde{\rho}(t) / \operatorname{Tr} \tilde{\rho}(t)$, where $t$ is the time since the last tunneling event. Each step of the simulation thus consists in determining the random waiting time using the CDF generated by Eq. (3), calculating the normalized density matrix at the end of the deterministic period and selecting the jump to be made.

\section{Results}

Using the formalism presented above we study the role of phonon-related processes in the system dynamics and in the measurement process. To this end, we perform stochastic simulations and compare the resulting system evolution in two cases: the realistic situation, with unobserved phonon scattering events, and the hypothetical one, with phonon scattering events treated as detection events. We are interested in three relevant quantities. One is the phase coherence of the system state, expressed by the off-diagonal element of the unconditional density matrix (that is, the density matrix averaged over very many realizations of the detection processes). This is in fact trivial, as it can be directly found from the Lindblad Eq. (1). The second quantity of interest is the degree of localization. Each single quantum trajectory representing the evolution of the conditional system state tends to one of the localized states, which corresponds to $z=\operatorname{Tr}\left(\rho \sigma_{z}\right) \rightarrow \pm 1$. In order to determine the average rate of this process one introduces the quantity $\left\langle z^{2}\right\rangle$ which represents the degree of localization of the electron in the DQD, where \langle\rangle denotes averaging over a large number of realizations. The third interesting quantity is the probability that the system state will be guessed correctly after it is measured for time $t$. Here we assume the following guessing procedure: for a given measurement outcome (number of tunneling events at the QPC) one decides that the electron is in the dot $i$ if the probability of getting this outcome with the electron in the dot $i$ is larger than the probability of the same outcome with the electron in the other dot. The probability $p$ of making the correct guess can easily be calculated from the Poisson distribution. A useful quantity, related to the amount of available information on the system state is the distinguishability of states $D=2 p-1$.

In Fig. 1, we show the dynamics of decoherence and localization for a certain set of parameters. In order to clarify the role of phonons in the system evolution from the point of view of both physics and information we compare the realistic case when the phonon scattering events play the role of noise and do not provide any information on the system state with the hypothetical case of "observable phonon scattering" in which the result of each scattering event is known. One should note that the physical processes of system-reservoir interactions are the same in both cases and only their informational aspect differs. If the phonon-related processes do not contribute to the observer's knowledge on the system state, they play no role in the measurement, hence they do not modify the localization dynamics which is related to the measurement (Fig. 1a). This changes if the phonon scattering is treated as a measurement process (Fig. 1b). On the other hand, whether phonon scattering events are observed or not is not relevant for the decoherence of the DQD, which reflects the correlations (entanglement) built between the system and its environment, irrespective of their informational content for the observer (Fig. 1c,d).

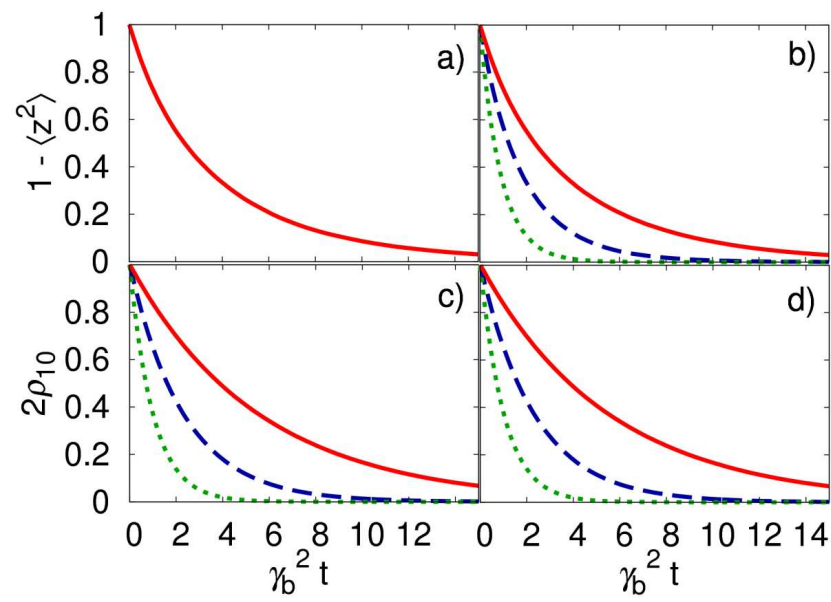

Fig. 1. Localization (a,b) and decoherence (c,d) due to simultaneous interaction of the system with the QPC and phonons. In $(\mathrm{a}, \mathrm{c})$ phonon scattering is treated as unobservable noise. In $(b, d)$ the information carried by phonon scattering events is treated as observable. Here $\gamma_{a} / \gamma_{b}=0.3$. Red solid line: $\gamma_{\mathrm{ph}}=0$ (no phonons), blue dashed line: $\gamma_{\mathrm{ph}}=0.5$, green dotted line: $\gamma_{\mathrm{ph}}=$ 0.9. In (a) all the three lines coincide. The results are averaged over $10^{5}$ realizations.

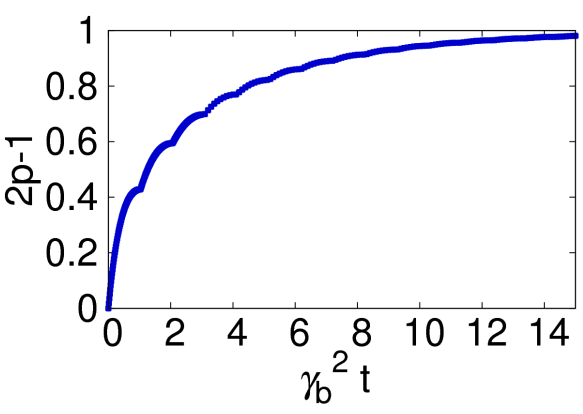

Fig. 2. The distinguishability of DQD states as a function of the measurement time for $\gamma_{a} / \gamma_{b}=0.3$. 
In Fig. 2, we show how the information available from the measurement process increases with time. By comparing with Fig. 1a, c, one can see that the time scale for this accumulation of information is the same as those of localization and of dephasing in the absence of phonons.

\section{Conclusion}

We have studied the weak measurement of charge states in a DQD performed by coupling the DQD to a QPC in the presence of additional phonon-induced dephasing. We have compared the three essential time scales relevant for the dynamics of the open system. We have shown that the rate of acquiring information on the system state is the same as the rate of localization, which is consistent with the measurement postulate of quantum mechanics. This rate is a lower limit for the dephasing rate, as extracting information must inevitably destroy quantum coherence. On the other hand, the dephasing rate is increased by the additional phonon-induced dephasing, while the two measurement-related rates are insensitive to the charge-phonon coupling.

\section{Acknowledgments}

This work was partly supported by the Czech Science Foundation under grant No. 202/07/J051.

\section{References}

[1] A.C. Johnson, J.R. Petta, J.M. Taylor, A. Yacoby, M.D. Lukin, C.M. Marcus, M.P. Hanson, A.C. Gossard, Nature 435, 925 (2005).

[2] T. Fujisawa, T. Hayashi, R. Tomita, Y. Hirayama, Science 312, 1634 (2006).

[3] T.M. Stace, S.D. Barrett, Phys. Rev. Lett. 92, 136802 (2004).

[4] A.N. Korotkov, Phys. Rev. B 60, 5737 (1999).

[5] A. Korotkov, D. Averin, Phys. Rev. B 64, 165310 (2001).

[6] H.-S. Goan, G. Milburn, Phys. Rev. B 64, 235307 (2001).

[7] H.-S. Goan, G. Milburn, H. Wiseman, H.B. Sun, Phys. Rev. B 63, 125326 (2001).

[8] D. Averin, E. Sukhorukov, Phys. Rev. Lett. 95, 126803 (2005).

[9] D. Averin, A. Korotkov, Phys. Rev. Lett. 94, 069701 (2005).

[10] P. Machnikowski, Phys. Rev. Lett. 96, 140405 (2006).

[11] J. Bylander, T. Duty, P. Delsing, Nature 434, 361 (2005).

[12] M.C. Cassidy, A.S. Dzurak, R.G. Clark, K.D. Petersson, I. Farrer, D.A. Ritchie, C.G. Smith, Appl. Phys. Lett. 91, 222104 (2007).

[13] T.M. Stace, S.D. Barrett, arXiv:cond-mat/0309610v2 (unpublished).

[14] H.-P. Breuer, F. Petruccione, The Theory of Open Quantum Systems, Oxford University Press, Oxford 2002. 